Corrigendum: Parallel states of pathological Wnt signaling in neonatal brain injury and colon cancer

Stephen P J Fancy, Emily P Harrington, Sergio E Baranzini, John C Silbereis, Lawrence R Shiow, Tracy J Yuen, Eric J Huang, Stavros Lomvardas \& David H Rowitch

Nat. Neurosci. 17, 506-512 (2014); published online 9 March 2014; corrected after print 30 July 2014

In the version of this article initially published, the wrong image was included as Figure $5 \mathrm{~g}$, top left panel. The image that appeared was the bottom left image from Supplementary Figure 9a; the correct image is the top right one from Supplementary Figure 9a. The error has been corrected in the HTML and PDF versions of the article.

Corrigendum: Nuclear BK channels regulate gene expression via the control of nuclear calcium signaling

Boxing Li, Wei Jie, Lianyan Huang, Peng Wei, Shuji Li, Zhengyi Luo, Allyson K Friedman, Andrea L Meredith, Ming-Hu Han, Xin-Hong Zhu \& Tian-Ming Gao

Nat. Neurosci. 17, 1055-1063 (2014); published online 22 June 2014; corrected after print 5 September 2014

In the version of this article initially published, item (ii) in the third paragraph of the Discussion read, "The ability to obtain high-resistance gigaohm seals during patch clamping in the nucleus-attached mode, when dozens of NPCs may be encompassed by the patch pipette, suggests that NPCs can exist in a closed state that restricts ion flow ${ }^{39}$ ". This sentence has been amended to cite its source, which the authors inadvertently eliminated while reducing the reference count for publication. That work has been added to the reference list as ref. 72: Matzke, A.J., Weiger, T.M. \& Matzke, M. Ion channels at the nucleus: electrophysiology meets the genome. Mol. Plant 3, 642-652 (2010). In addition, the text has been reworded to avoid directly quoting from the source without attribution; the revised text reads, "Patch clamping in the nucleus-attached mode puts many NPCs in the tip of a patch pipette. The high-resistance seal between the pipette and NE suggests that NPCs can shift to a closed state that restricts ion flux ${ }^{39,72}$." The error has been corrected in the HTML and PDF versions of the article.

\title{
Corrigendum: miR-92a regulates expression of synaptic GluA1-containing AMPA receptors during homeostatic scaling
}

Mathieu Letellier, Sara Elramah, Magali Mondin, Anaïs Soula, Andrew Penn, Daniel Choquet, Marc Landry, Olivier Thoumine \& Alexandre Favereaux

Nat. Neurosci. 17, 1040-1042 (2014); published online 13 July 2014; corrected after print 4 August 2014

In the version of this article initially published, the e-mail address for author O.T. was given as othoumin@u-bordeaux.fr and a protein in the last paragraph of the main text was given as SVA2. These should be, respectively, olivier.thoumine@u-bordeaux.fr and SV2A. The errors have been corrected in the HTML and PDF versions of the article.

\section{Corrigendum: The GABAergic parafacial zone is a medullary slow wave sleep-promoting center}

Christelle Anaclet, Loris Ferrari, Elda Arrigoni, Caroline E Bass, Clifford B Saper, Jun Lu \& Patrick M Fuller Nat. Neurosci. 17, 1217-1224 (2014); published online 17 August 2014; corrected after print 28 August 2014

In the version of this article initially published, the colors of the data points were reversed relative to the key in Figure $3 \mathrm{~d}$, left panel. The error has been corrected in the HTML and PDF versions of the article. 